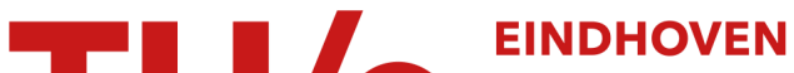 UNIVERSITY OF TECHNOLOGY
}

\section{Trapping of neutral atoms with resonant microwave radiation}

Citation for published version (APA):

Agosta, C. C., Silvera, I. F., Stoof, H. T. C., \& Verhaar, B. J. (1989). Trapping of neutral atoms with resonant microwave radiation. Physical Review Letters, 62(20), 2361-2364. https://doi.org/10.1103/PhysRevLett.62.2361

DOI:

10.1103/PhysRevLett.62.2361

Document status and date:

Published: 01/01/1989

\section{Document Version:}

Publisher's PDF, also known as Version of Record (includes final page, issue and volume numbers)

\section{Please check the document version of this publication:}

- A submitted manuscript is the version of the article upon submission and before peer-review. There can be important differences between the submitted version and the official published version of record. People interested in the research are advised to contact the author for the final version of the publication, or visit the $\mathrm{DOI}$ to the publisher's website.

- The final author version and the galley proof are versions of the publication after peer review.

- The final published version features the final layout of the paper including the volume, issue and page numbers.

Link to publication

\section{General rights}

Copyright and moral rights for the publications made accessible in the public portal are retained by the authors and/or other copyright owners and it is a condition of accessing publications that users recognise and abide by the legal requirements associated with these rights.

- Users may download and print one copy of any publication from the public portal for the purpose of private study or research.

- You may not further distribute the material or use it for any profit-making activity or commercial gain

- You may freely distribute the URL identifying the publication in the public portal.

If the publication is distributed under the terms of Article $25 \mathrm{fa}$ of the Dutch Copyright Act, indicated by the "Taverne" license above, please follow below link for the End User Agreement:

www.tue.nl/taverne

Take down policy

If you believe that this document breaches copyright please contact us at:

openaccess@tue.nl

providing details and we will investigate your claim. 


\title{
Trapping of Neutral Atoms with Resonant Microwave Radiation
}

\author{
Charles C. Agosta and Isaac F. Silvera \\ Lyman Laboratory of Physics, Harvard University, Cambridge, Massachusetts 02138
}

H. T. C. Stoof and B. J. Verhaar

Department of Physics, Eindhoven University of Technology, 5600 MB Eindhoven, The Netherlands

(Received 19 December 1988)

\begin{abstract}
We discuss a resonant microwave trap for neutral atoms. Because of the long spontaneous radiation time this trap is remarkably different from the optical trap. It also has advantages over static magnetic traps that trap the excited spin state of the lowest electronic level, in that atoms predominantly in the spin ground state can be trapped. We analyze the relaxation-ejection lifetime of atoms in such a trap using the formalism of dressed atomic states. Results are applied to atomic hydrogen and the possibility of Bose-Einstein condensation is considered.
\end{abstract}

PACS numbers: $32.80 . \mathrm{Pj}, 42.50 . \mathrm{Vk}, 67.65 .+\mathrm{z}$

The past several years have been witness to the exciting development of trapping of neutral atoms with optical radiation from a laser source ${ }^{1}$ or with static magnetic fields. ${ }^{2-4}$ In this Letter we show that a gas of atoms can be trapped using forces derived from the gradient of a microwave radiation field on resonance with an atomic transition. ${ }^{5}$ The principal difference between microwave and optical traps is the virtual absence of microwave spontaneous emission. This changes the nature of the trap potential and eliminates the spontaneous emission heating of the atoms. In comparison to the static magnetic trap the microwave trap has the primary advantage of being able to trap atoms with a ground-state character. Because the static magnetic trap utilizes a field minimum, ${ }^{6}$ it is limited to trapping of atoms in the ground electronic state with excited spin states, which spin relax to the ground state at a density-dependent rate. These lower-state atoms are then ejected from the trap, ${ }^{7}$ resulting in low densities of trapped atoms. In contrast, the microwave trap can operate with either a field maximum or minimum and the trapped atoms will be an admixture of the excited and the ground states, depending on the detuning of the radiation from resonance. We study the lifetime of the atomic density and show that it can be much longer in a microwave trap than in the static trap and thus much higher densities are possible. This is important in efforts to obtain Bose-Einstein condensation (BEC) in atomic hydrogen, which is discussed as a specific example.

We first derive the trap potential. ${ }^{8}$ We shall treat the atom as a two-level system and denote the states by $|e\rangle$ and $|g\rangle$ for excited and ground states. A very simple approach for calculating the depth of a magnetic trap is the dressed-atom approach used for optical traps by Dalibard and Cohen-Tannoudji. ${ }^{9,10}$ An atom in a singlemode radiation field with magnetic operator $\mathbf{B}_{R}$ can be described by the following Hamiltonian,

$$
\mathscr{H}=H_{R}+H_{\text {atom }}-\mu \cdot \mathbf{B}_{R}(\mathbf{r}) \text {. }
$$

Here, $H_{R}=\hbar \omega a^{\dagger} a$ is the Hamiltonian of the radiation field with photon angular frequency $\omega$ and creation and annihilation operators $a^{\dagger}$ and $a$, respectively. $H_{\text {atom }}$ $=p^{2} / 2 m+\hbar \omega_{0} b^{\dagger} b$ is the Hamiltonian of the atom of mass $m$ with the two levels separated in energy by $\hbar \omega_{0}$, where $b^{+}$and $b$ are the atomic raising and lowering operators for the levels $|e\rangle$ and $|g\rangle$. We have assumed that the atom interacts with a magnetic field $\mathbf{B}=\mathbf{B}_{0}+\mathbf{B}_{R}$ through its magnetic dipole moment $\boldsymbol{\mu} . \mathbf{B}_{0}$ is a static field and is included in $H_{\text {atom. If there is no zero-field }}$ splitting of the atom, the resonance frequency is $\omega_{0}=\mu_{B} B_{0} / \hbar$, where $\mu_{B}$ is the Bohr magneton. The last term in Eq. (1) is the interaction of the atom with the microwave field which, including resonant terms only, is given by $-\boldsymbol{\mu}_{e g} \cdot \lambda \mathscr{B}(\mathbf{r})\left(a^{\dagger} b+a b^{\dagger}\right)$, where $\boldsymbol{\mu}_{e g}$ is a magnetic dipole transition-matrix element and $\lambda$ is the polarization vector.

If the interaction between the field and the atom is zero then the eigenstates of this Hamiltonian fall into manifolds separated by energy $\hbar \omega$. Each manifold consists of a pair of states $|g, N\rangle$ and $|e, N-1\rangle$ split by $\hbar \delta$, where $N$ is the photon occupation number and $\delta=\omega-\omega_{0}$ is the detuning. When the coupling term is included, the dressed states are ${ }^{9}$

$$
\begin{aligned}
& |1 N \mathbf{r}\rangle \equiv|1\rangle=\cos \theta(\mathbf{r})|e, N-1\rangle+\sin \theta(\mathbf{r})|g, N\rangle, \\
& |2 N \mathbf{r}\rangle \equiv|2\rangle=-\sin \theta(\mathbf{r})|e, N-1\rangle+\cos \theta(\mathbf{r})|g, N\rangle,
\end{aligned}
$$

where the angle $\theta(\mathbf{r})$ is defined by $\cos 2 \theta(\mathbf{r})=-\delta / \Omega(\mathbf{r})$ and $\sin 2 \theta(\mathbf{r})=\omega_{r}(\mathbf{r}) / \Omega(\mathbf{r})$. Here, $\omega_{r}=\boldsymbol{\mu}_{e g} \cdot \lambda 2 \sqrt{N}$ $\times \mathcal{B}(\mathbf{r}) / \hbar$ is the Rabi frequency, and $\Omega(\mathbf{r})=\left[\omega_{r}^{2}(\mathbf{r})\right.$ $\left.+\delta^{2}\right]^{1 / 2}$. The corresponding energies are $E_{i N}(\mathbf{r})$ $=N \hbar \omega+E_{i}(\mathbf{r})$ with

$$
\begin{aligned}
& E_{1}(\mathbf{r})=-\hbar \delta / 2+\hbar \Omega(\mathbf{r}) / 2, \\
& E_{2}(\mathbf{r})=-\hbar \delta / 2-\hbar \Omega(\mathbf{r}) / 2 .
\end{aligned}
$$

These energy levels vary with position in an inhomogeneous field through the dependence of the Rabi frequency on $\mathbf{r}$.

The depth of the trap is the difference in energy for an atom in the microwave field and its energy in zero field. 
For a microwave field with a maximum, state $|2\rangle$ is a trapping state with potential

$$
U_{t}=\hbar / 2\left[|\delta|-\left(\omega_{r}^{2}+\delta^{2}\right)^{1 / 2}\right] .
$$

State $|1\rangle$ is a barrier state or an antitrapping state with $U_{\text {at }}=-U_{t}$. The maximum trap depth is at $\delta=0$, yielding $U_{t \max } / k_{B}=\hbar \omega_{r} / 2 k_{B}=0.47 B_{R}$ in kelvins with $B_{R}$ in teslas. Here we have taken $\mu_{\text {eg }} \cdot \lambda=\sqrt{2} \mu_{B}$ and have used circularly polarized microwave radiation; for linearly polarized radiation the depth is reduced by $\sqrt{2}$. The microwave trap has the interesting property that $U_{t}$ has its maximum depth for $\delta=0$ and $|2\rangle$ remains a trapping state for both positive and negative values of $\delta$. Moreover, as can be seen from the states in Eq. (2b), the nature of $|2\rangle$ changes from predominantly $|e, N-1\rangle$ to $|g, N\rangle$ as $\delta$ goes from positive to negative values. In Figs. 1(a) and 1(b), we plot the trap potential for the two dressed states as a function of the detuning $\delta$, as well as the dependence of the admixture of states on detuning.

We contrast these results with the optical laser trap where the excited state is separated from the ground state by an optical interval. This has a potential well ${ }^{9,11}$

$$
U_{\mathrm{opt}}=(\hbar / 2) \delta \ln \left(1+\omega_{r}^{2} / 2 \delta^{2}\right),
$$

also plotted in Fig. 1(a) for the same interaction as in Eq. (1). In this trap $U_{\text {opt }}=0$ for $\delta=0$ and the potential changes from trapping to antitrapping as $\delta$ changes sign. This remarkable change of nature from the microwave case is caused by the short spontaneous emission lifetime characteristic of electric dipoles in the optical region (of order $10^{-8} \mathrm{~s}$ ) which couple the dressed manifolds. By contrast, electron-spin magnetic dipole transitions have spontaneous-emission lifetimes of several million years. In an optical trap, an atom makes many transitions between dressed states in the time required to move across the trap and the force is an average found by multiplying the time spent in each state times the force in each state, resulting in Eq. (5). For $\delta=0$ the dressed states are equal admixtures of $|e, N-1\rangle$ and $|g, N\rangle$ so that the time spent in each state is equal, thus the force is zero. In the microwave case there is virtually no spontaneous emission; in addition it is easy to show that nonadiabatic transition rates are negligible. For these reasons the states of the atoms remain unchanged in time. This results in a deeper potential as a function of $\delta$ [see Fig. 1(a)]; it also precludes spontaneous emission heating which plaques the optical trap. Still, to create a useful well depth, large-amplitude microwave fields are needed. They can be attained in a cavity such as a concentric resonator; the application of such a resonator to the microwave trap has been discussed elsewhere. ${ }^{8}$

Unlike the above traps, the static mágnetic trap, which has a depth $\left|U_{t}\right|=\left|\mu_{B} \Delta B_{0}\right|$, can be made much deeper with a large static gradient $\Delta B_{0}$ on the order of $1 \mathrm{~T}$. However, the trapped atoms must be in state $|e\rangle$ since a
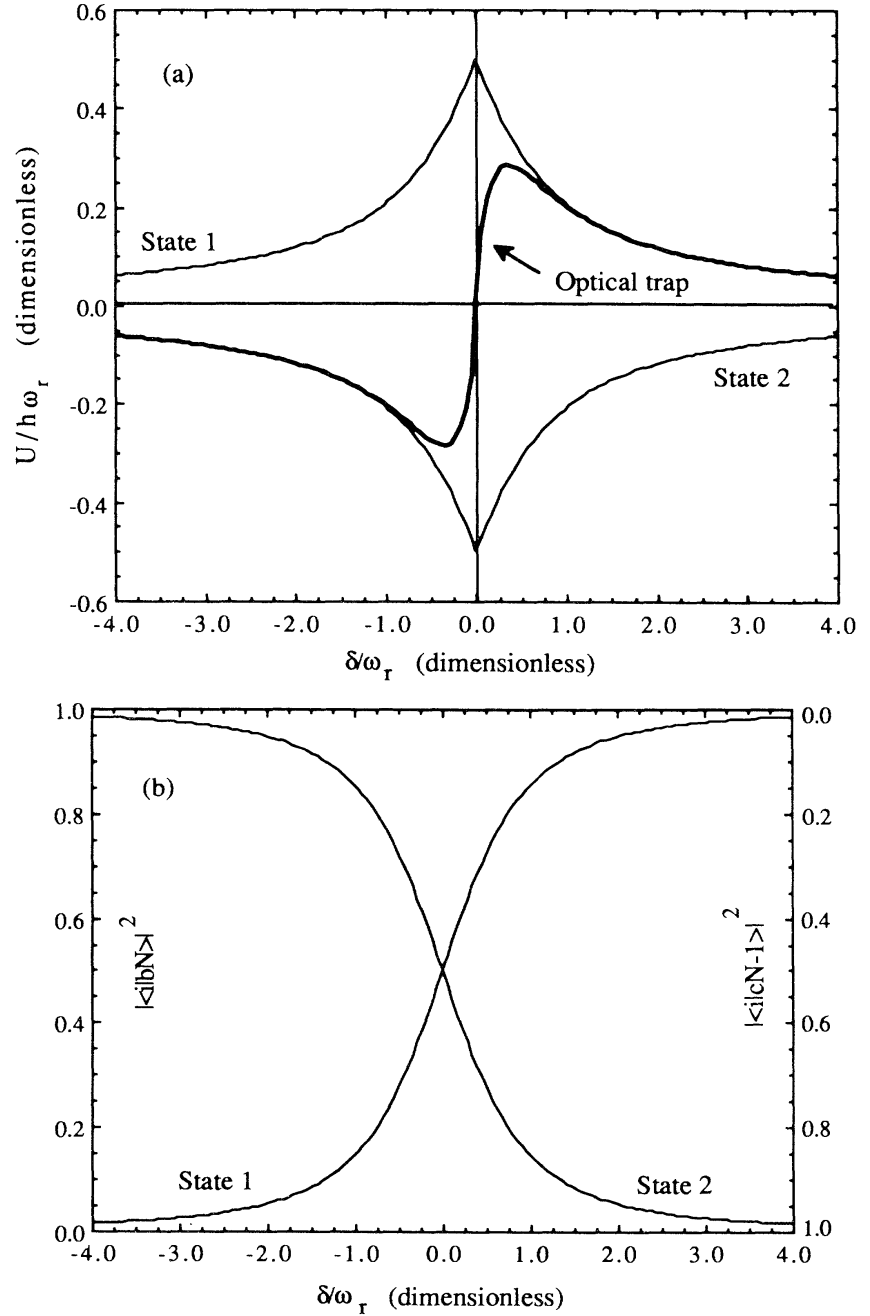

FIG. 1. (a) Potential depth for the two dressed atom states compared to the depth for an optical trap (with the same interaction) as a function of $\delta / \omega_{r}$. (b) The probabilities for the bare states as admixed into the trapping and antitrapping states, as a function of $\delta / \omega_{r}$.

static trap can only be made with a field maximum. Because of density-dependent collisional relaxation to state $|g\rangle$, which is an antitrapping state (atoms are ejected), the density in this trap rapidly decays down to a relatively low value.

A crucial consideration of the viability of the microwave trap is its lifetime for decay of the density due to collisional relaxation. Although this trap can be applied to any species with an effective two-level system, we shall consider atomic hydrogen, and compare it to the existing static trap. Hydrogen has four hyperfine states enumerated $|a\rangle,|b\rangle,|c\rangle$, and $|d\rangle$, from low to high energy. In a high field, electron-spin resonance is allowed between states $|b\rangle$ and $|c\rangle$ (as well as $|a\rangle$ and $|d\rangle$ ). We shall focus on the $|b\rangle$ and $|c\rangle$ states as a two-level 
system, modifying our earlier notations so that $|g\rangle \equiv|b\rangle$ and $|e\rangle \equiv|c\rangle$. Since the dressed state $|2\rangle$ is an admixture of $|b\rangle$ and $|c\rangle,|2\rangle-|2\rangle$ binary collisions can result in the transition of $|c\rangle$ to $|b\rangle$ during a collision. A transition to a $b$ state results in the formation of state $|1\rangle$ or |2) with a calculable probability. Since those atoms in state $|1\rangle$ are ejected, the density decays. Collisional relaxation between bare states $|b\rangle$ and $|c\rangle$ have been studied earlier ${ }^{7}$ and are a result of spin-exchange or magnetic dipole-dipole interactions. Both $T_{1}$ (inelastic relaxation) and $T_{2}$ (dephasing of the transverse spin) processes can lead to decay of the density of atoms in state $|2\rangle$. We find that the $T_{1}$ processes correspond to relaxation between dressed-atom manifolds, whereas the $T_{2}$ processes occur within a single manifold and have the unusual property of being thermally activated. This can be understood by considering states $|2\rangle$ and $|1\rangle$ in the rotating coordinate system of the rf magnetic field. An isolated atom in the state $|2\rangle$ has its fictitious spin $\frac{1}{2}$ pointing along the effective field, whereas $|1\rangle$ points opposite. A $T_{2}$ collision will shift the transverse component of the spin leading to a superposition of states $|1\rangle$ and $|2\rangle$ and thus to decay by ejection. In a preliminary consideration of relaxation in the microwave trap, ${ }^{8} T_{2}$ events were not considered and some coherent contributions to $T_{1}$ were omitted. Here we consider both channels of relaxation using a $T$-matrix formalism [Eq. (7), below]. Our detailed considerations will be focused on hydrogen, but the concepts are applicable to other species such as sodium, and we shall first present a general treatment.

Outside the range of the interatomic interaction two bare atoms interact only with the radiation field. In this situation the combined system of photons and atoms can be described by the symmetrized and normalized states $|\{i j\} N\rangle$ :

$$
\begin{aligned}
& |\{11\} N\rangle=\cos ^{2} \theta|\{e e\}, N-2\rangle+(1 / \sqrt{2}) \sin 2 \theta|\{e g\}, N-1\rangle+\sin ^{2} \theta|\{g g\}, N\rangle, \\
& |\{12\} N\rangle=-(1 / \sqrt{2}) \sin 2 \theta|\{e e\}, N-2\rangle+\cos 2 \theta|\{e g\}, N-1\rangle+(1 / \sqrt{2}) \sin 2 \theta|\{g g\}, N\rangle, \\
& |\{22\} N\rangle=\sin ^{2} \theta|\{e e\}, N-2\rangle-(1 / \sqrt{2}) \sin 2 \theta|\{e g\}, N-1\rangle+\cos ^{2} \theta|\{g g\}, N\rangle .
\end{aligned}
$$

The energies are $E_{i j N}=N \hbar \omega+E_{i}+E_{j}$ [cf. Eq. (3)]. These states may be interpreted as the free states of two indistinguishable dressed atoms and correspond to the possible initial and final channels of a scattering process. ${ }^{12}$ The relaxation rates for the processes $i j N \rightarrow i^{\prime} j^{\prime} N^{\prime}$ are found from a $T$-matrix calculation ${ }^{13}$

$$
G_{i j N \rightarrow i^{\prime} j^{\prime} N^{\prime}}(T)=4 \pi^{3} \hbar^{2} \mu\left\langle p_{i^{\prime} j^{\prime}} \sum_{l^{\prime} m^{\prime}} \sum_{l m}\left|T_{l^{\prime} m^{\prime}\left\{i^{\prime} j^{\prime}\right\} N^{\prime}, \operatorname{lm}\{i j\} N}\left(p_{i^{\prime} j^{\prime}, p_{i j}}\right)\right|^{2}\right\rangle_{\mathrm{th}},
$$

where $\mu$ is the reduced mass, $p_{i j}$ and $p_{i^{\prime} j^{\prime}}$ are the magnitude of the initial and final relative momenta, respectively, and the subscript th implies a thermal average. Note that to find the decay of the atomic density inside the trap we should, in addition to the thermal average over initial momenta $\mathbf{p}_{i j}$, average over the number of photons. However, in an intense radiation field we can neglect fluctuations and replace $N$ by its average value $\bar{N}$ in Eq. (7). The relevant decay rates are then $G_{22 \bar{N} \rightarrow i^{\prime} j^{\prime} N^{\prime}}(T)$.

To evaluate these quantities it is important to point out that the central (singlet or triplet) interaction between two bare hydrogen atoms couples only the states $|\{i j\} N\rangle$ with the same value of $N$. Since the energy splitting $\hbar \Omega$ within this manifold is very small compared to the strength of the central interaction, the degenerate-internal-states (DIS) approximation ${ }^{13}$ is valid and used throughout the following. Note that the dipolar transitions among states with the same $N$ are completely suppressed by the centrifugal barrier in either the initial or final state.

If $N^{\prime}=\bar{N}$ all processes are endothermal. In order to find the dominant low-temperature behavior of the rates we use the relation ${ }^{13}$

$$
G_{22 \bar{N} \rightarrow i^{\prime} j^{\prime} \bar{N}}(T)=G_{i^{\prime} j^{\prime} \bar{N} \rightarrow 22 \bar{N}}(T) \exp \left(-\Delta_{i^{\prime} j^{\prime}} / k_{B} T\right),
$$

expand the rate constant for the inverse (exothermal) reaction, and take the lowest order $k_{B} T / \Delta_{i^{\prime} j^{\prime}}$, where $\Delta_{i^{\prime} j^{\prime}}=\hbar \Omega\left(\delta_{i^{\prime}, 1}+\delta_{j^{\prime}, 1}\right)$ denotes the energy difference between the final and initial channels. This expansion is found by applying the DIS approximation to the $T$ matrix in Eq. (7). Doing so, we find that transitions within a manifold are associated with elastic exchange collisions between bare atoms and are at an effective rate defined by $d n_{2} / d t=-G_{T_{2}}^{\text {eff }} n_{2}^{2}$. For the hydrogen atom

$$
G_{T_{2}}^{\mathrm{eff}}=2.3 \times 10^{-13}\left(\hbar \Omega / k_{B}\right)^{1 / 2} \sin ^{2} 2 \theta\left[B_{C}^{4} /\left(B_{0}^{2}+B_{C}^{2}\right)^{2}\right]\left[\sin ^{4} \theta \exp \left(-\hbar \Omega / k_{B} T\right)+\sqrt{2} \exp \left(-2 \hbar \Omega / k_{B} T\right)\right]
$$

in units of $\mathrm{cm}^{3} / \mathrm{s}$ with the static field $B_{0}$ in tesla and the constant $B_{C}=0.05064 \mathrm{~T}$ originating from a consideration of the hyperfine levels in the field $B_{0}$.

To obtain the above expression, one has to evaluate the difference $T_{00\{c c\}, 00\{c c\}}(0,0)-T_{01}(0,0)$, where $T_{l s}(0,0)$ is a (on-shell) $T$-matrix element for singlet or triplet scattering at zero energy. Applying the DIS approximation once again, but now to bare atom states, this difference can be expressed in terms of scattering lengths $a^{(s)}$. In this second DIS approximation, we assume the hyperfine states $|\{c c\}\rangle,|\{b d\}\rangle,|\{a c\}\rangle$, and $|\{a a\}\rangle$ to be degenerate, similar to the 
same assumption for the dressed states $|\{i j\} N\rangle$ within a manifold. The result is

$$
\begin{aligned}
& T_{00\{c c\}, 00\{c\}}(0,0)-T_{01}(0,0) \\
& \quad=\left[B_{C}^{2} /\left(B_{0}^{2}+B_{C}^{2}\right)\right]\left(2 \pi \hbar m_{H}\right)^{-1}\left(a^{(0)}-a^{(1)}\right) .
\end{aligned}
$$

Equation (10) is fully confirmed by a rigorous coupledchannel calculation. A numerical evaluation of Eq. (9) shows that in the experimental circumstances envisaged, i.e., strong magnetic fields of order $5 \mathrm{~T}$, the $T_{2}$ processes are unimportant compared to $\left(T_{1}\right)$ transitions between different manifolds which are due mainly to dipolar interactions and will be considered next.

Dipolar relaxation caused by the dominant electronelectron magnetic interaction $V^{d}$ is possible for both $N^{\prime}=\bar{N}-1$ and $\bar{N}-2$. Because this weak interaction can be treated as a small perturbation, we have

$$
\begin{aligned}
& T_{l^{\prime} m^{\prime}\left\{i^{\prime} j^{\prime}\right\} N^{\prime}, \operatorname{lm}\{i j\} N}\left(p_{i^{\prime} j^{\prime}}, p_{i j}\right) \\
& ={ }_{c}\left\langle\psi_{l}\left(\mathbf{I}^{\prime}{ }^{\prime}{ }_{\left.i i^{\prime} j^{\prime}\right\} N^{\prime}}\left|V^{d}\right| \psi_{\operatorname{lm}}^{(+)}\{i j\}_{N}\right\rangle_{c},\right.
\end{aligned}
$$

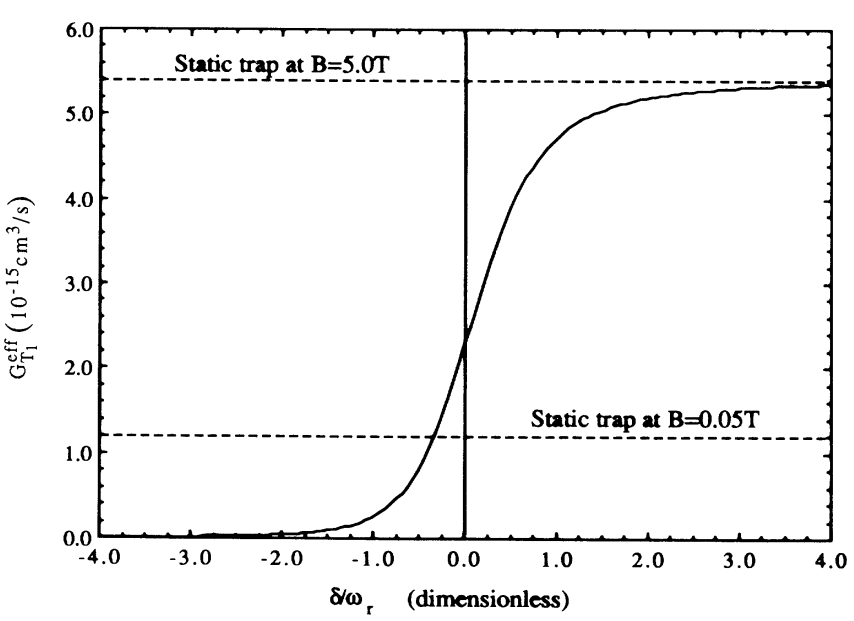

FIG. 2. Decay-rate constant of hydrogen atoms in the microwave trap as a function of $\delta / \omega_{r}$. We compare to the static trap for magnetic fields of 0.05 and $5 \mathrm{~T}$.

using the notation of Ref. 13. The initial and final distorted wave functions incorporate the central interaction to all orders and can be approximated by neglecting the energy splitting within the respective manifolds $|\{i j\} N\rangle$ and $\left|\left\{i^{\prime} j^{\prime}\right\} N^{\prime}\right\rangle$. Furthermore, if we make use of the fact that at high magnetic fields the hyperfine state $|\{c c\}\rangle$ has a dominant triplet character, we get

$$
G_{T_{1}}^{\text {eff }}(B, T)=\sin ^{4} \theta\left[4 \sin ^{2} 2 \theta+\left(1-4 \cos ^{2} \theta\right)^{2}\right] G_{c c \rightarrow b c}(B, T)+2 \sin ^{4} \theta\left(\sin ^{4} \theta+\frac{1}{4} \sin ^{2} 2 \theta\right) G_{c c \rightarrow b b}(B, T) .
$$

Here $G_{T_{1}}^{\text {eff }}$ is defined locally by $d n_{2} / d t=-G_{T_{1}}^{\text {eff }} n_{2}^{2}$.

Because of the phase relations between interfering $T$ matrix elements, the rate (12) could be expressed in the rates $G_{c c \rightarrow b c}\left(=G_{b c \rightarrow b b}\right)$ and $G_{c c \rightarrow b b}$ for collisions between bare hydrogen atoms, which have been calculated previously in Refs. 7 and 13. Note that from these papers we find a justification for the neglect of the $|a\rangle$ and $|d\rangle$ hyperfine states in the above treatment. At magnetic field strengths of the order of $5 \mathrm{~T}$, the dominant relaxation processes in a gas of atoms populating the $b$ and $c$ states are $c c \rightarrow b b, c c \rightarrow b c$, and $b c \rightarrow b b$. From these results we find that the half-life of the trapped atoms is $\tau=\left(G_{T_{1}}^{\text {eff }} n_{0}\right)^{-1}$, where $n_{0}$ is the initial trapped density. In Fig. 2 we plot $G_{T_{1}}^{\text {eff }}$ as a function of $\theta$, as well as $G^{\text {eff }}$ for the static trap. ${ }^{7}$ We see that by detuning to small $\theta$ so that $|2\rangle \simeq|g, N\rangle$ the microwave trap can be made much more stable than the static trap. The resulting potentially higher starting densities overcome the difficulties of attaining BEC, addressed by Tommila. ${ }^{14}$ Evaporative cooling ${ }^{15}$ of the trapped atoms as a means of attaining BEC can be easily accomplished by detuning, which lowers the well depth.

We would like to thank W. Phillips, H. Metcalf, C. Cohen-Tannoudji, and E. Eliel for useful discussions. Support has been provided by DOE Grant No. DEFG02-85ER45190 and NATO Collaborative Research Grant No. 0543/88.
'S. Chu, J. E. Bjorkholm, A. Ashkin, and A. Cable, Phys. Rev. Lett. 57, 314 (1986).

${ }^{2}$ A. L. Migdall, J. V. Prodan, W. D. Phillips, T. H. Bergeman, and H. J. Metcalf, Phys. Rev. Lett. 54, 2596 (1985).

${ }^{3}$ H. F. Hess, G. P. Kochanski, J. M. Doyle, N. Masuhara, D. Kleppner, and T. J. Greytak, Phys. Rev. Lett. 59, 672 (1987).

${ }^{4}$ R. van Roijen, J. J. Berkhout, S. Jaakkola, and J. T. M. Walraven, Phys. Rev. Lett. 61, 931 (1988).

${ }^{5}$ M. Bloom and K. Erdman, Can. J. Phys. 40, 179 (1962); M. Bloom, E. Enga, and H. Lew, Can. J. Phys. 45, 1481 (1967); R. M. Hill and T. F. Gallagher, Phys. Rev. A 12, 451 (1975).

${ }^{6}$ W. H. Wing, Prog. Quantum Electron. 8, 181 (1984).

${ }^{7}$ A. Lagendijk, I. F. Silvera, and B. J. Verhaar, Phys. Rev. B 33, 626 (1986).

${ }^{8}$ C. C. Agosta and I. F. Silvera, in Proceedings of the International Conference on Spin-Polarized Quantum Systems, Torino, Italy, June 1988 (to be published).

${ }^{9}$ J. Dalibard and C. Cohen-Tannoudji, J. Opt. Soc. Am. B 2, 1707 (1985).

${ }^{10}$ W. D. Phillips (private communication).

${ }^{11}$ J. P. Gordon and A. Ashkin, Phys. Rev. A 21, 1606 (1980).

12P. S. Julienne, Phys. Rev. Lett. 61, 698 (1988).

${ }^{13}$ H. T. C. Stoof, J. M. V. A. Koelman, and B. J. Verhaar, Phys. Rev. B 38, 4688 (1988).

${ }^{14}$ T. Tommila, Europhys. Lett. 2, 789 (1986).

${ }^{15}$ H. F. Hess, Phys. Rev. B 34, 3476 (1986). 